\title{
Downregulation of circ-PSMB6 suppresses NSCLC progression and metastasis through sponging miR- 532-5p and regulating EZH1 expression
}

Mingming Jin ( $\sim$ asdjinmingming@126.com )

Shanghai University of Medicine and Healthy Science

Junqian Zhang

Shanghai University of Medicine and Health Sciences

Yue Wu

Shanghai University of Medicine and Health Sciences

Yitian Dai

Shanghai University of Medicine and Health Sciences

Gang Huang

Shanghai University of Medicine and Health Sciences

Research

Keywords: non-small cell lung cancer (NSCLC), circ-PSMB6, miR-532-5p, EZH1, cancer stem cells

Posted Date: April 9th, 2021

DOI: https://doi.org/10.21203/rs.3.rs-392149/v1

License: (9) This work is licensed under a Creative Commons Attribution 4.0 International License.

Read Full License 


\section{Abstract}

Background: Accumulating reports showed how circular RNAs (circRNAs) act importantly during tumor progression via regulating gene expression, but regulatory mechanisms remain largely unknown. Current investigation clarified circRNA regulatory mechanisms in non-small cell lung cancer (NSCLC).

Methods: High-throughput sequencing and quantitative reverse transcription polymerase chain reaction (RT-qPCR) detection were utilized to explore circRNA expression in NSCLC tissues and cells. Our lab did statistical analyses and luciferase reporter analysis to validate correlations between circRNA, miRNA and gene expression. We transfected NSCLC cells with different vectors, and transwell migration, Cell Counting Kit-8 (CCK-8) proliferation along with colony formation assays were performed. In vivo tumorigenesis and metastasis assays were utilized to validate the circRNA role in NSCLC.

Results: Data illustrated that hsa_circ_0041595 (circ-PSMB6) incremented in NSCLC cell lines and tissues, while circ-PSMB6 downregulation suppressed NSCLC cell proliferation and invasion in vitro and in vivo. Bioinformatics analysis and luciferase reporter data verified that miR-532-5p and Enhancer Of Zeste 1 Polycomb Repressive Complex 2 Subunit (EZH1) were circ-PSMB6 downstream targets in NSCLC cells. Overexpression of EZH1 or miR-532-5p inhibition reversed NSCLC cell invasion and proliferation after silencing circ-PSMB6. Further experiments discovered that circ-PSMB6 can influence cancer stem cell differentiation by regulating miR-532-5p/EZH1.

Conclusions: Taken together, we found that circ-PSMB6 suppressed NSCLC metastasis and progression via sponging miR-532-5p and regulating EZH1 expression.

\section{Background}

Lung cancer is identified by high morbidity and poor prognosis. NSCLC is the main class of lung cancer, which accounts for about $85 \%$ lung cancer cases [1]. We now know that smoking is the predominant reason for NSCLC [2]. Since NSCLC shows no early apparent clinical symptoms and screening applications are not efficient, majority patients diagnosed with NSCLC are in an advanced stage with poor prognosis [3]. Many clinical investigations have shown that metastasis hinders NSCLC cancer treatment. Therefore, a better understanding the mechanisms underlying metastasis is crucial for treating NSCLC effectively.

Circular RNAs (circRNAs) are a class of endogenous and conserved non-coding RNA (ncRNA) that form covalently closed continuous loops via back-splicing with no $3^{\prime}$ or $5^{\prime}$ end [4]. More and more studies discovered a substantial effect of ncRNAs including circRNAs, long ncRNAs and microRNAs (miRNAs) on tumor development $[5,6]$. For example, circFGFR1 increments progression and anti-PD-1 resistance through sponging miR-381-3p in NSCLC cells [7]. The circ-ABCB10 promotes NSCLC proliferation and inhibits cell apoptosis by repressing KISS1 [8]. The circGFRA1 promotes NSCLC progression through miR188-3p sponging [9]. Whereas circRNA roles in NSCLC progression are still unclear. Our study was thus aimed at verifying the potential effects and underlying mechanisms of circRNAs in NSCLC progression. 


\section{Methods \& Materials}

\section{Tissue samples}

We collected six fresh NSCLC tissues along with paired adjacent noncancerous lung tissues. We obtained consent from patients in Renji Hospital at Shanghai Jiaotong University. We evaluated pathological and histological diagnostics of NSCLC based upon Revised International System for Staging Lung Cancer. Our lab snap-froze samples in liquid nitrogen and maintained them at $-80^{\circ} \mathrm{C}$ before RNA extraction. Ethics Committee of Shanghai University of Medicine and Health Sciences supervised this study.

\section{Strand-specific RNA-Seq library preparation and next-generation RNA-Seq}

Total RNA from 3 paired NSCLC tissues along with adjacent noncancerous lung tissue was extracted by TRIzol reagent (Invitrogen, Carlsbad, CA, USA). Our team prepared 3 $\mu \mathrm{g}$ total RNA from each sample using VAHTS Total RNA-seq (H/M/R) Library Prep Kit from Illumina (Vazyme Biotech Co. Ltd., Nanjing, China) to erase ribosomal RNA retaining other classes of RNA such as ncRNA and mRNA. Our lab dealt RNA with RNase R (Epicenter, $40 \mathrm{U}, 37^{\circ} \mathrm{C}$ for $3 \mathrm{~h}$ ), followed by TRIzol purification. Technician made RNAseq libraries through KAPA Stranded RNA-Seq Library Prep Kit (Roche, Basel, Switzerland) and subjected them to deep sequencing leveraging the Illumina HiSeq 4000 at Aksomics, Inc., Shanghai, China.

\section{Ethics statement}

We purchased BALB/c nude mice with four weeks old weighing 15 20 g from Shanghai SLAC Laboratory Animal Co. Ltd. (Shanghai, China). Ethics Committee in Shanghai University of Medicine and Health Sciences of Shanghai supervised the procedures and our team conducted the experiments following their guidelines. Our lab performed all surgical procedures under anesthesia and tried to eliminate suffering. Our team anesthetized the mice with an intraperitoneal injection of $30 \mathrm{mg} / \mathrm{kg}$ sodium pentobarbital.

\section{Cell culture}

Our lab obtained NSCLC human pulmonary epithelial cell lines H1299, PC9, H1650, A549 and H1975 and control non-tumorigenic bronchial epithelium BEAS-2B cell line from American Type Culture Collection (Manassas, VA, USA). We cultured cell lines in Dulbecco's Modified Eagle's medium (Gibco, Grant Island, NY, USA) which was supplied with $10 \%$ fetal bovine serum in humidified atmosphere with $5 \% \mathrm{CO}_{2}$ at $37^{\circ} \mathrm{C}$.

\section{RNA interference and overexpression}

miR-532-5p inhibitors and siRNA against circ-PSMB6 (si-circ-PSMB6) were obtained from GenePharma (Shanghai, China). We transferred cells to 6-well culture plates and transfected them via Lipofectamine 2000 (Invitrogen, Carlsbad, CA, USA) following the protocol. To induce Enhancer of Zeste 1 Polycomb Repressive Complex 2 Subunit (EZH1) overexpression, we transfected cells with a pCDNA3.0 vector. For 
xenograft experiments, a lentiviral-mediated circ-PSMB6-silencing vector was transfected into PC9 cells (sh-circ-PSMB6).

\section{Total RNA isolation and RT-qPCR}

Our lab employed TRIzol reagent to obtain total RNA from tumor cells and tissues following manufacturer's protocol. Our lab measured RNA sample concentration and purity at absorbances of 230, 260, and $280 \mathrm{~nm}$ with NanoDrop ND-1000 spectrophotometer (Thermo Fisher Scientific, Wilmington, USA). We regarded $\mathrm{OD}_{260} / 280$ and $\mathrm{OD}_{260} / 230$ ratios in $1.8 \sim 2.1$ and $>1.8$ as acceptable.

We used SuperScript II Reverse Transcriptase (Thermo Fisher Scientific) to reverse-transcribe RNA ( $1 \mu \mathrm{g})$ into cDNA. RT-qPCR was performed with an ABI 7300 Real-Time System (Applied Biosystems, Foster City, CA, USA) using primer pairs synthesized by GenePharma specific for glutathione synthetase (GSS), glutathione peroxidase 4 (GPX4) and glyceraldehyde 3-phosphate dehydrogenase (GAPDH), and TaqMan Universal PCR Master Mix (Thermo Fisher Scientific). Technician quantified gene expression by $2^{-\Delta \Delta C t}$ method. Primers applied to assay circ-PSMB6 expression were forward, 5》-GAGATGCCCTGTACTGATGC$3 \rrbracket$ and reverse, $5 \rrbracket-C T T T C T C C T C C G G T A C T G C-3 \rrbracket$. The miR-532-5p primers were forward, 5囚CGGCCATGCCTTGAGTGTA-3囚 and reverse, 5囚-GCAGGGTCCGAGGTATTC-3囚. The EZH1 primers were

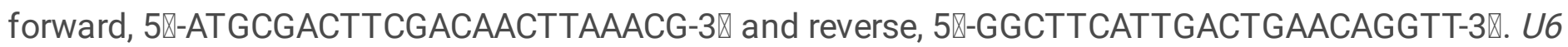

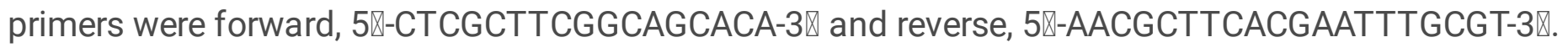
GAPDH primers were forward, 5囚-GCACCGTCAAGGCTGAGAAC-3\ and reverse, 5囚GGATCTCGCTCCTGGAAGATG-3】.

\section{Fluorescence in situ hybridization (FISH)}

A probe specific to circ-PSMB6 (Dig-5'-GTTTGTCACAGAGAAACAGTTGCTTTGAG-3'-Dig) was made by Geneseed Biotech (Guangzhou, China). Our lab detected signals via Cy3-conjugated anti-digoxin and antibiotin antibodies (Jackson ImmunoResearch Inc., West Grove, USA). Our team counterstained nuclei with 4,6-diamidino-2-phenylindole (DAPI) and captured figures with Zeiss LSM 700 confocal microscope (Carl Zeiss, Oberkochen, Germany).

\section{Bioinformatics analyses}

We predicted the correlations between circRNA, miRNA and mRNA using the websites at http://starbase.sysu.edu.cn/, http://www.circbank.cn/ and https://circinteractome.nia.nih.gov/.

\section{Transwell migration assay}

Our lab assessed cell migration through Costar ${ }^{\circledR}$ Transwell $^{\circledR}$ cell culture inserts (Corning Inc., Corning, NY, USA) following instructions. After incubating the cells for $1 \mathrm{~d}$, our lab erased cells on Transwell chamber upper surfaces via cotton swabs. Technician fixed cells on lower surfaces in methanol for 10 minutes and stained them with Crystal Violet. Our team counted and photographed cells in 5 fields that randomly selected. 


\section{Plate colony formation assay}

PC9 and A549 cells after different treatments were resuspended and counted, and we seeded 200 cells/well in plates with six wells. Our lab incubated cells for 2 weeks, with the medium changed every 3 days. We took photographs with a fluorescence microscope before ending the experiment, then washed the cells twice with PBS. The cells were stained in $500 \mu \mathrm{L}$ Giemsa dye for 10-20 min, washed three times with $\mathrm{ddH}_{2} \mathrm{O}$ and photographed with a digital camera.

\section{CCK-8 assay}

We determined proliferation rates 1, 2, and 3 days after transfection using Cell Counting Kit-8 (CCK-8) assays following instructions. Technician incubated cells in 10\% CCK-8 solution diluted in normal culture medium at $37^{\circ} \mathrm{C}$ until visual color conversion occurred. We read every well absorbance using microplate reader at $570 \mathrm{~nm}$.

\section{Dual-luciferase reporter assay}

Putative miR-532-5p binding sites in target gene EZH1 and in circ-PSMB6 were cloned into the psi-CHECK vector (Promega, Madison, Wisconsin, USA). Wild type (Wt) or mutated (Mut) 3'-UTR sequences from EZH1 or Wt and Mut miR-532-5p binding sites from circ-PSMB6 were inserted downstream from firefly luciferase as the primary luciferase signal and were designated EZH1-Wt/circ-PSMB6-Wt and EZH1Mut/circ-PSMB6-Mut. psi-CHECK vector itself supplied a Renilla luciferase signal as a normalization factor to compensate for the differences between transfection and harvested efficiencies. Our team then transfected 293T cells with these constructs using Lipofectamine 2000 and detected Renilla and firefly luciferase activities $1 \mathrm{~d}$ after transfection with Dual-Luciferase Reporter Assay System (Promega) utilizing luminometer (Molecular Devices, U.S.A.). We processed relative Renilla luciferase activity following the manufacturer instructions.

\section{Tumor sphere formation assays}

PC9 and A549 cells were harvested and resuspended as single cells in serum-free medium. Following cell counting, our lab seeded 200 cells/well in $200 \mu \mathrm{L}$ serum-free medium in plates with 96 wells, 10 wells/group, altering medium every $2 \mathrm{~d}$. Our lab obtained five images from randomly selected regions in each well group using camera-equipped microplate reader (Leica, Wetzlar, Germany) and computed sphere percent as number of spheres/200.

\section{Cell cycle detection}

Our team dissociated cells in logarithmic growth phase with $0.25 \%$ trypsin, resuspended them in PBS and fixed them in ice-cold $70 \%$ ethyl alcohol overnight at $4^{\circ} \mathrm{C}$. Our team centrifuged cells at $1000 \mathrm{rpm}$ for five minutes, resuspended them in $50 \mu \mathrm{L}$ RNase $A$ and incubated them at $37^{\circ} \mathrm{C}$ for half of an hour. We added $400 \mu \mathrm{L}$ propidium iodide (PI) to suspension for $0.5 \mathrm{~h}$, followed by flow cytometry (BD Bioscience, CA, USA). 


\section{Animal studies}

To detect the circ-PSMB6 role in lung cancer metastasis model, we intravenously injected $1 \times 10^{6}$ stable lentiviral-mediated circ-PSMB6-silenced PC9 cells (sh-circ-PSMB6) or negative control (NC) PC9 cells into male nude mice (Chinese Science Academy, Shanghai, China) via tail vein. After one month, our team analyzed PC9 cell metastasis through bioluminescence imaging following intravenous luciferin injection (150 mg luciferin/kg body weight) into tails.

For xenograft assays, our team subcutaneously injected $1 \times 10^{6}$ modified or control PC9 cells (sh-circPSMB6 or $\mathrm{Wt}$,) into the male nude mice right flank. Tumor volumes were calculated as $0.5 \times$ length $\times$ width $^{2}$ at time points indicated, and technician excised all tumors $4 \mathrm{w}$ after injection. All animals were treated according to instructions from Animal Care Committee in Shanghai University of Medicine and Health Sciences.

\section{Immunohistochemical analysis}

Our team fixed tumor tissue samples in $10 \%$ formalin solution, embedded them in paraffin and sliced them into $5-\mu \mathrm{m}$-thick sections, which we stained with antibodies against Ki67 to validate cell proliferation, using AxioPhot light microscope (Carl Zeiss AG, Oberkochen, Germany) equipped with digital camera

\section{Statistical analyses}

Statistician compared differences between any 2 groups by unpaired or paired 2-tailed $t$-test. Bioinformatician leveraged Pearson's correlation coefficient to characterize correlations between groups. Bioinformatician denoted data as means \pm standard deviation. Our team considered probability $(p)$ values $<0.05$ statistically significant. We employed GraphPad Prism (GraphPad Software, La Jolla, CA, USA) for statistical analyses.

\section{Results}

\section{Differential expression of circRNAs in NSCLC.}

High-throughput sequencing found that 1308 circRNAs were upregulated and 1530 circRNAs were downregulation in NSCLC tissues (T) comparing to adjacent non-tumorous tissues ( $T$ ), shown as volcano plots in Fig. 1A. The length distribution of circRNAs were mostly concentrated below 1,000 bp (Fig. 1B). We selected the 10 most upregulated circRNAs for further study (Fig. 1C). RT-qPCR results validated that only hsa_circ_0041595 (circ-PSMB6) expression was upregulated significantly in NSCLC tissues $(n=6)$ comparing to adjacent non-tumorous tissues (Fig. 1D). FISH revealed circ-PSMB6 expression in NSCLC tissues which was mainly localized to the cytoplasm (Fig. 1E).

circ-PSMB6 expression increased in NSCLC cells and circ-PSMB6 knockdown caused cell cycle arrest in G2 phase. 
Bioinformatics analyses indicated that hsa_circ_0041595 was located in chr17:4699456-4701790 and originated from an exon in the gene encoding Proteasome 20S Subunit Beta (PSMB6) containing 826 bp. Therefore, hsa_circ_0041595 was named circ-PSMB6 (Fig. 2A). RT-qPCR assays showed that circ-PSMB6 expression in NSCLC cells lines PC9, H1299, A549, H1650 and H1975 was increased comparing to BEAS2B control cells. Because PC9 and A549 cells had the highest circ-PSMB6 expression levels, which were chosen for the study (Fig. 2B). We constructed siRNA against circ-PSMB6 and found that circ-PSMB6 expression was significantly decreased when circ-PSMB6 was silenced in both PC9 and A549 cells (Fig. 2C). Flow cytometric analysis showed that circ-PSMB6 silencing caused cell cycle arrest in the G2 phase (Fig. 2D), suggesting that circ-PSMB6 plays a role in NSCLC progression.

\section{Downregulating circ-PSMB6 inhibited cell metastasis and proliferation in vivo and in vitro.}

CCK8 (Fig. 3A-B) and clonal formation assays (Fig. 3C and 3D) demonstrated that circ-PSMB6 downregulation inhibited cell proliferation in PC9 and A549 cells. In nude mouse xenografts, circ-PSMB6 silencing suppressed PC9 tumor growth in both volume and weight (Fig. 3E-G). Immunohistochemistry also illustrated that circ-PSMB6 downregulation inhibited Ki67 expression. These studies thus demonstrated that circ-PSMB6 downregulation inhibited tumor growth and cell proliferation both in vivo and in vitro.

Transwell assays were used to examine PC9 and A549 cell migratory capacity. Results indicated that circPSMB6 silencing suppressed cell migration (Fig. 4A and 4B). Live imaging showed the circ-PSMB6 effect on NSCLC metastasis regarding PC9 cells 4 weeks after tail intravenous injection. circ-PSMB6 silencing inhibited the number of metastatic foci in lung tissues based on hematoxylin/eosin staining (Fig. 4C-E).

\section{EZH1 and miR-532-5p were the downstream targets of circ-PSMB6.}

Bioinformatics analysis showed that circ-PSMB6 could interact with a series of miRNAs. A comprehensive Venn diagram analysis utilizing the Starbase, circBank and circlnteractome databases found that only miR-532-5p and miR-1179 could interact with circ-PSMB6 in a common region that was identified by all three databases (Fig. 5A). RT-qPCR analyses demonstrated that miR-532-5p expression decremented significantly in NSCLC tissues (Fig. 5B). We then constructed a luciferase reporter vector containing Wt or Mut circ-PSMB6 sequence and co-transfected it with miRNA mimics into 293T cells. Data illustrated that only miR-532-5p could decrease fluorescein intensity significantly, advising that miR532-5p was the circ-PSMB6 downstream target (Fig. 5C). Luciferase reporter data further confirmed that miR-532-5p inhibited luciferase activity in Wt constructs, yet not in Mut cell lines (Fig. 5D), demonstrating that miR-532-5p was the circ-PSMB6 target.

Bioinformatics analyses also indicated that EZH1 was miR-532-5p downstream target. To better verify relationship between miR-532-5p and EZH1, our team constructed Mut and Wt 3'-UTR-EZH1 sequences containing miR-532-5p binding sequence into luciferase reporter vector (Fig. 5E), which was transfected into 293T cells with or not miR-532-5p mimic. Luciferase reporter data indicated that miR-532-5p 
inhibited luciferase activity in Wt cells, yet not in Mut cell lines (Fig. 5F), suggesting that EZH1 was the miR-532-5p target.

RT-qPCR data demonstrated that circ-PSMB6 silencing suppressed circ-PSMB6 expression, while treatment with miR-532-5p inhibitor or overexpressing EZH1 did not affect circ-PSMB6 expression in either PC9 and A549 cells (Fig. 5G and 5H). These results advised that both miR-532-5p and EZH1 were circ-PSMB6 downstream. RT-qPCR data found that circ-PSMB6 silencing increased miR-532-5p expression, while EZH1 overexpression did not affect circ-PSMB6-induced miR-532-5p expression (Fig. $5 \mathrm{I}$ and $5 \mathrm{~J}$ ), informing that miR-532-5p is downstream from circ-PSMB6. Our results also revealed that circPSMB6 silencing decreased EZH1 expression, whereas downregulation of miR-532-5p reversed circPSMB6 inhibitory effect on EZH1 expression. After transfection with EZH1 overexpression vector, the expression of EZH1 was significantly increased (Fig. 5K and 5L), suggesting that circ-PSMB6 promoted EZH1 expression by sponging miR-532-5p.

\section{Overexpression of EZH1 or miR-532-5p inhibition reversed NSCLC cell migration and proliferation after silencing circ-PSMB6 via differential regulation of cancer stem cells.}

CCK8 and clonal formation assays showed that overexpression of EZH1 or inhibition of miR-532-5p reversed proliferation of both PC9 and A549 cells after circ-PSMB6 was silenced (Fig. 6A-E). Transwell assays to assess cell migration also showed that overexpressing of EZH1 or inhibiting miR-532-5p blocked the migratory capacity of both PC9 and A549 cells after silencing circ-PSMB6 (Fig. 6F-H). Western blots indicated that overexpression of EZH1 or inhibition of miR-532-5p slowed tumor sphere formation by both PC9 and A549 cells after circ-PSMB6 was silenced (Fig. 7), suggesting that these effects were mediated by differential regulation of cancer stem cells.

\section{Discussion}

The circRNAs belong to a class of recently discovered ncRNAs, and a number of reports show that they may be applied as diagnostic biomarkers as well as therapeutic targets in various cancers. circ_0078767 suppresses NSCLC through protecting RASSF1A expression through sponging of miR-330-3p [10]. circNDUFB2 inhibits NSCLC progression by destabilizing IGF2BPs and activating anti-tumor immunity [11]. The present investigation discovered that circ-PSMB6 expression incremented in NSCLC tissues comparing with non-tumorous tissues. circ-PSMB6 downregulation arrested cell cycles of A549 and PC9 cells in $\mathrm{G} 2$ phase, suppressed cell proliferation and slowed invasion in in vitro and in vivo experiments. These data demonstrated that circ-PSMB6 may facilitate the progression of NSCLC.

More and more studies suggested that circRNAs can act as miRNA sponges to regulate gene expression $[12,13]$. In this study, we found that circ-PSMB6 interacted with miR-532-5p, which was confirmed by luciferase report analysis. Previous research has revealed that miR-532-5p enhances breast cancer migration and proliferation via targeting RERG [14] and suppresses lung cancer cell invasion and migration via inhibiting CCR4 [15]. miR-532-5p is also prognostic marker which suppresses cell invasion and proliferation through targeting TWIST1 in epithelial ovarian cancer [16]. The present study found that 
downregulation of circ-PSMB6 promoted miR-532-5p expression, while miR-532-5p inhibition restored invasive and proliferative capacity of NSCLC cells when circ-PSMB6 was silenced.

Further results indicated that miR-532-5p could act with EZH1 3'UTR and suppress EZH1 expression at mRNA level. Luciferase reporter experiments demonstrated that EZH1 was miR-532-5p target.

Downregulating miR-532-5p restored EZH1 expression after circ-PSMB6 was silenced. Overexpressing EZH1 reversed the decrease in proliferation and invasion after downregulation of circ-PSMB6. The zeste (EZH) 2 enhancer functions importantly in NSCLC progression. EZH2 functions critically in tumorigenesis and cancer progression via epigenetic gene silencing and chromatin remodeling $[17,18]$. More reports validated that $\mathrm{EZH} 2$ overexpression occurs in various human malignancies such like breast, bladder, esophageal, oral, hepatocellular, gastric, colon, prostate, and endometrial cancers [19-21]. Elevated EZH2 expression has been correlated with advanced stages of ovarian cancer [22]. Our current investigation suggested that EZH1 overexpression or miR-532-5p inhibition reversed NSCLC cell proliferation and migration after circ-PSMB6 was silenced via the differential regulation of cancer stem cells.

\section{Conclusion}

In summary, our study demonstrated that downregulation of circ-PSMB6 expression decreased NSCLC cell proliferation and invasions via miR-532-5p/EZH2 signaling regulation. The results inferred that circPSMB6 is potential diagnostic biomarker of NSCLC, and suggested that drugs targeting circ-PSMB6 may play a promising role in NSCLC treatment.

\section{Declarations}

\section{Ethics approval and consent to participate}

None.

\section{Consent for publication}

All authors have agreed to publish this manuscript.

\section{Availability of data and material}

The data generated or analyzed during this study are included in this article, or if absent are available from the corresponding author upon reasonable request.

\section{Competing interests}

The authors declare that they have no competing interests.

\section{Funding}


The study was funded by National Natural Science Foundation of China (nos. 81830052, 82003142, and 81530053), the Shanghai Key Laboratory of Molecular Imaging (no. 18DZ2260400) and the Special Innovative Training Program for Postgraduates (no. Y2020077).

\section{Author Contributions}

MJ, JZ, and YW performed research and analyzed results. YD discussed results. MJ edited the paper. MJ and GH designed the research and drafted the paper. GH conceived the study. All authors approved the final manuscript.

\section{Acknowledgements}

Not applicable.

\section{Abbreviations}

NSCLC, non-small cell lung cancer; circRNAs, Circular RNAs; FISH, fluorescence in situ hybridization; CCK8, Cell Counting Kit-8; DAPI, 4,6-diamidino-2-phenylindole; miRNA, microRNA; ceRNAs, competitive endogenous RNAs; WT, wild-type; MUT, mutated.

\section{References}

1. Sung H, Ferlay J, Siegel RL, Laversanne M, Soerjomataram I, Jemal A, Bray F. Global cancer statistics 2020: GLOBOCAN estimates of incidence and mortality worldwide for 36 cancers in 185 countries. CA Cancer J Clin 2021.

2. Perez-Moreno P, Brambilla E, Thomas R, Soria JC. Squamous cell carcinoma of the lung: molecular subtypes and therapeutic opportunities. Clin Cancer Res. 2012;18:2443-51.

3. Crino L, Cappuzzo F. Present and future treatment of advanced non-small cell lung cancer. Semin Oncol. 2002;29:9-16.

4. Reck M. What future opportunities may immuno-oncology provide for improving the treatment of patients with lung cancer? Ann Oncol. 2012;23(Suppl 8):viii28-34.

5. Anastasiadou E, Jacob LS, Slack FJ. Non-coding RNA networks in cancer. Nat Rev Cancer. 2018;18:5-18.

6. Liu B, Ye B, Yang L, Zhu X, Huang G, Zhu P, Du Y, Wu J, Qin X, Chen R, Tian Y, Fan Z. Long noncoding RNA IncKdm2b is required for ILC3 maintenance by initiation of Zfp292 expression. Nat Immunol. 2017;18:499-508.

7. Zhang PF, Pei X, Li KS, Jin LN, Wang F, Wu J, Zhang XM. Circular RNA circFGFR1 promotes progression and anti-PD-1 resistance by sponging miR-381-3p in non-small cell lung cancer cells. Mol Cancer. 2019;18:179.

8. Zheng JP, Dai YM, Chen Z, Chen Q, Zheng Y, Lin X, Cui TJ. Circular RNA circ-ABCB10 promotes nonsmall cell lung cancer proliferation and inhibits cell apoptosis through repressing KISS1. Eur Rev 
Med Pharmacol Sci. 2020;24:2518-24.

9. Yao J, Xu G, Zhu L, Zheng H. circGFRA1 Enhances NSCLC Progression by Sponging miR-188-3p. Onco Targets Ther. 2020;13:549-58.

10. Chen T, Yang Z, Liu C, Wang L, Yang J, Chen L, Li W. Circ_0078767 suppresses non-small-cell lung cancer by protecting RASSF1A expression via sponging miR-330-3p. Cell Prolif. 2019;52:e12548.

11. Li B, Zhu L, Lu C, Wang C, Wang H, Jin H, Ma X, Cheng Z, Yu C, Wang S, Zuo Q, Zhou Y, Wang J, Yang $C$, Lv Y, Jiang L, Qin W. circNDUFB2 inhibits non-small cell lung cancer progression via destabilizing IGF2BPs and activating anti-tumor immunity. Nat Commun. 2021;12:295.

12. Zhang F, Zhang R, Zhang X, Wu Y, Li X, Zhang S, Hou W, Ding Y, Tian J, Sun L, Kong X. Comprehensive analysis of circRNA expression pattern and circRNA-miRNA-mRNA network in the pathogenesis of atherosclerosis in rabbits. Aging. 2018;10:2266-83.

13. Yang G, Zhang Y, Yang J. Identification of Potentially Functional CircRNA-miRNA-mRNA Regulatory Network in Gastric Carcinoma using Bioinformatics Analysis. Med Sci Monit. 2019;25:8777-96.

14. Huang L, Tang $X$, Shi X, Su L. miR-532-5p promotes breast cancer proliferation and migration by targeting RERG. Exp Ther Med. 2020;19:400-8.

15. Hu J, Wang L, Guan C. MiR-532-5p Suppresses Migration and Invasion of Lung Cancer Cells Through Inhibiting CCR4. Cancer Biother Radiopharm. 2020;35:673-81.

16. Wei H, Tang QL, Zhang K, Sun JJ, Ding RF. miR-532-5p is a prognostic marker and suppresses cells proliferation and invasion by targeting TWIST1 in epithelial ovarian cancer. Eur Rev Med Pharmacol Sci. 2018;22:5842-50.

17. Simon JA, Lange CA. Roles of the EZH2 histone methyltransferase in cancer epigenetics. Mutat Res. 2008;647:21-9.

18. Cao R, Zhang Y. The functions of E(Z)/EZH2-mediated methylation of lysine 27 in histone H3. Curr Opin Genet Dev. 2004;14:155-64.

19. Matsukawa Y, Semba S, Kato H, Ito A, Yanagihara K, Yokozaki H. Expression of the enhancer of zeste homolog 2 is correlated with poor prognosis in human gastric cancer. Cancer Sci. 2006;97:484-91.

20. Bryant RJ, Cross NA, Eaton CL, Hamdy FC, Cunliffe VT. EZH2 promotes proliferation and invasiveness of prostate cancer cells. Prostate. 2007;67:547-56.

21. Collett K, Eide GE, Arnes J, Stefansson IM, Eide J, Braaten A, Aas T, Otte AP, Akslen LA. Expression of enhancer of zeste homologue 2 is significantly associated with increased tumor cell proliferation and is a marker of aggressive breast cancer. Clin Cancer Res. 2006;12:1168-74.

22. Rizzo S, Hersey JM, Mellor P, Dai W, Santos-Silva A, Liber D, Luk L, Titley I, Carden CP, Box G, Hudson $\mathrm{DL}$, Kaye SB, Brown R. Ovarian cancer stem cell-like side populations are enriched following chemotherapy and overexpress EZH2. Mol Cancer Ther. 2011;10:325-35.

\section{Figures}


A

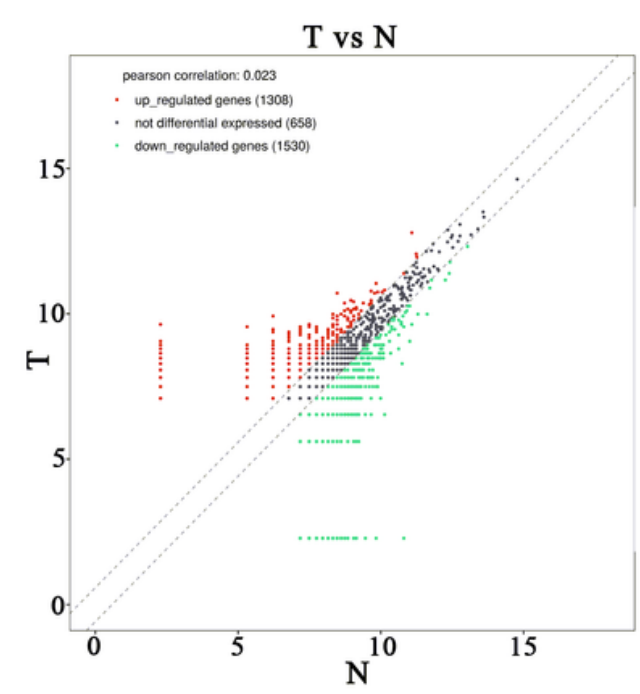

D

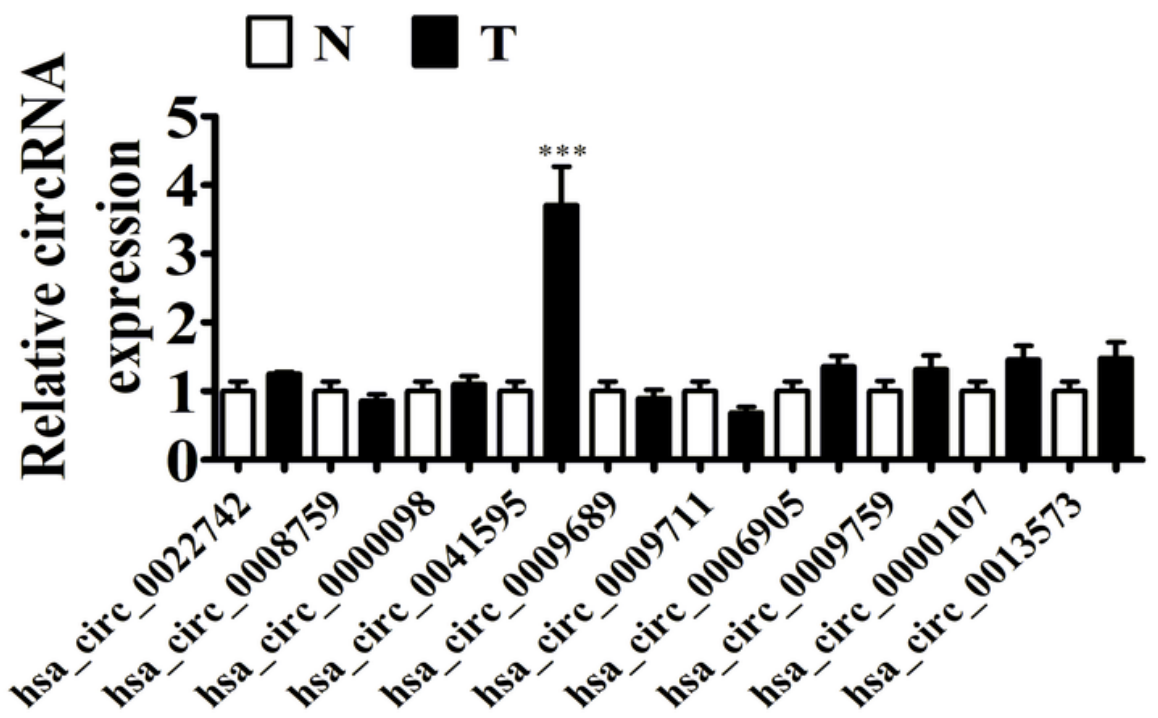

B

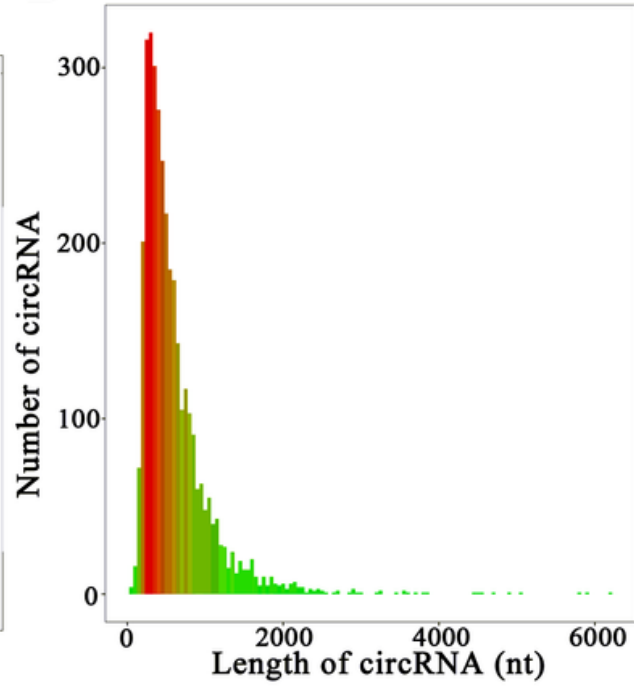

$\mathrm{E}$

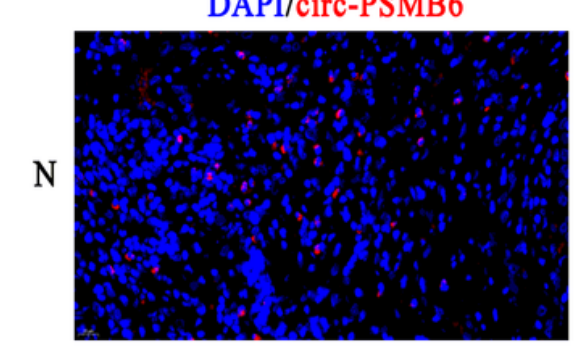

$\mathrm{T}$

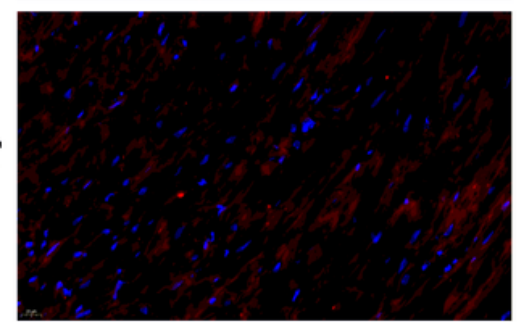

\section{Figure 1}

The diffeerent expression of circRNA in non-small cell lung cancer (NSCLC). (A) Volcano plots are using for visualizing differential expression between two different groups. $x$-axis: log2 ratio of circRNA expression levels between normal and tumor tissues. $y$-axis: the false discovery rate value (-log10 transformed) of circRNAs. (B) Length distribution of the identified circRNAs. $x$-axis: the length of circRNAs detected in this study. $y$-axis: the abundance of circRNAs classified by different lengths. (C) Heat map of all differentially expressed circRNAs between normal and tumor tissues. (D) Relative expression of the ten indicated circRNAs from NSCLC tumor tissues and adjacent non-tumor tissues listed in measured by RtqPCR. The data are presented as the mean \pm SD. ${ }^{* \star} \mathrm{p}<0.001 \mathrm{vs}$. (E and F) Fluorescence in situ hybridization (FISH) detection show the expression and subcellular localization of circ-PSMB6 in NSCLC tumor tissues and adjacent non-tumor tissues. N, non-tumor tissues; T, tumor tissues. 
chr17:4699456-4701790

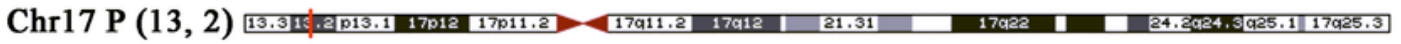
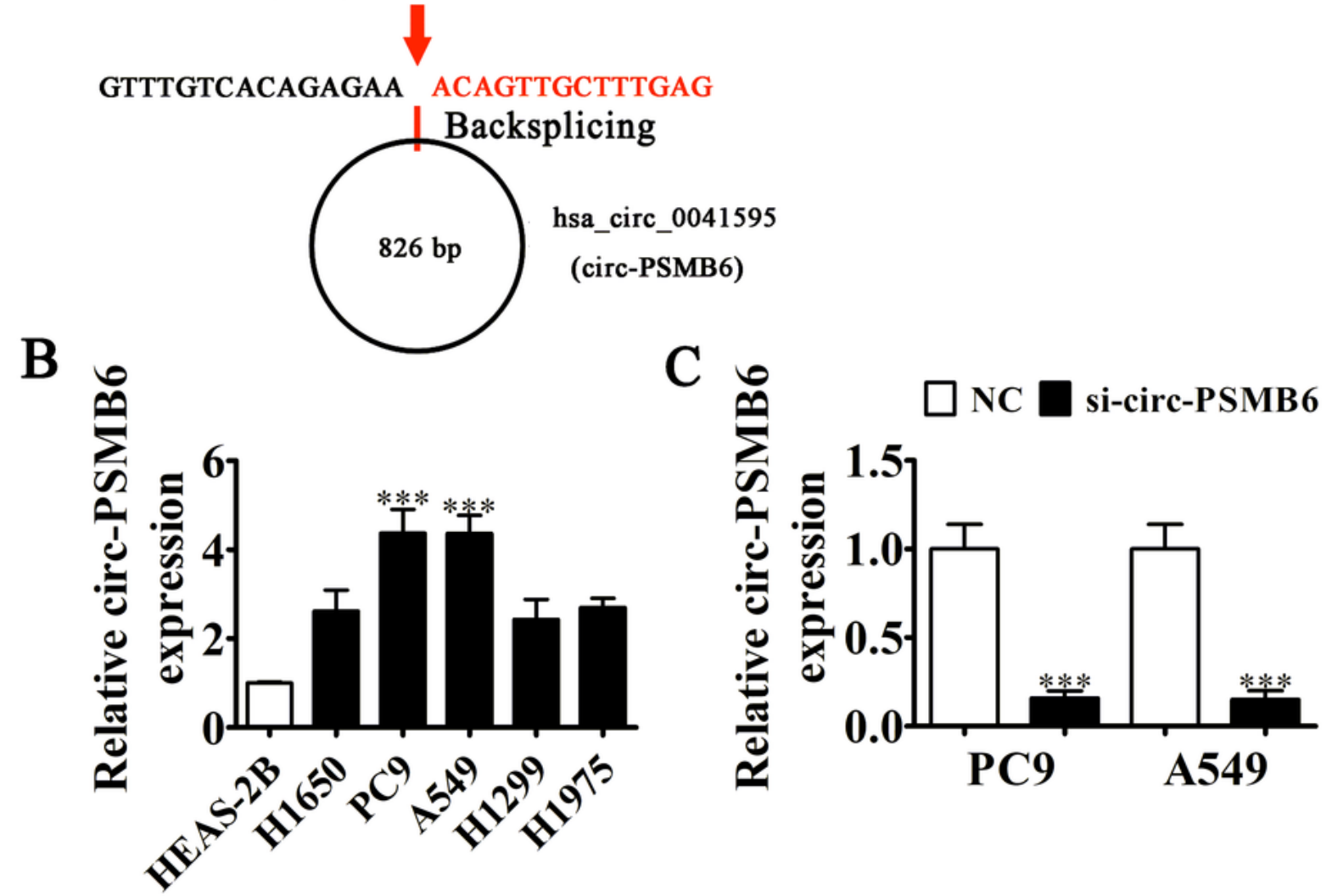

D
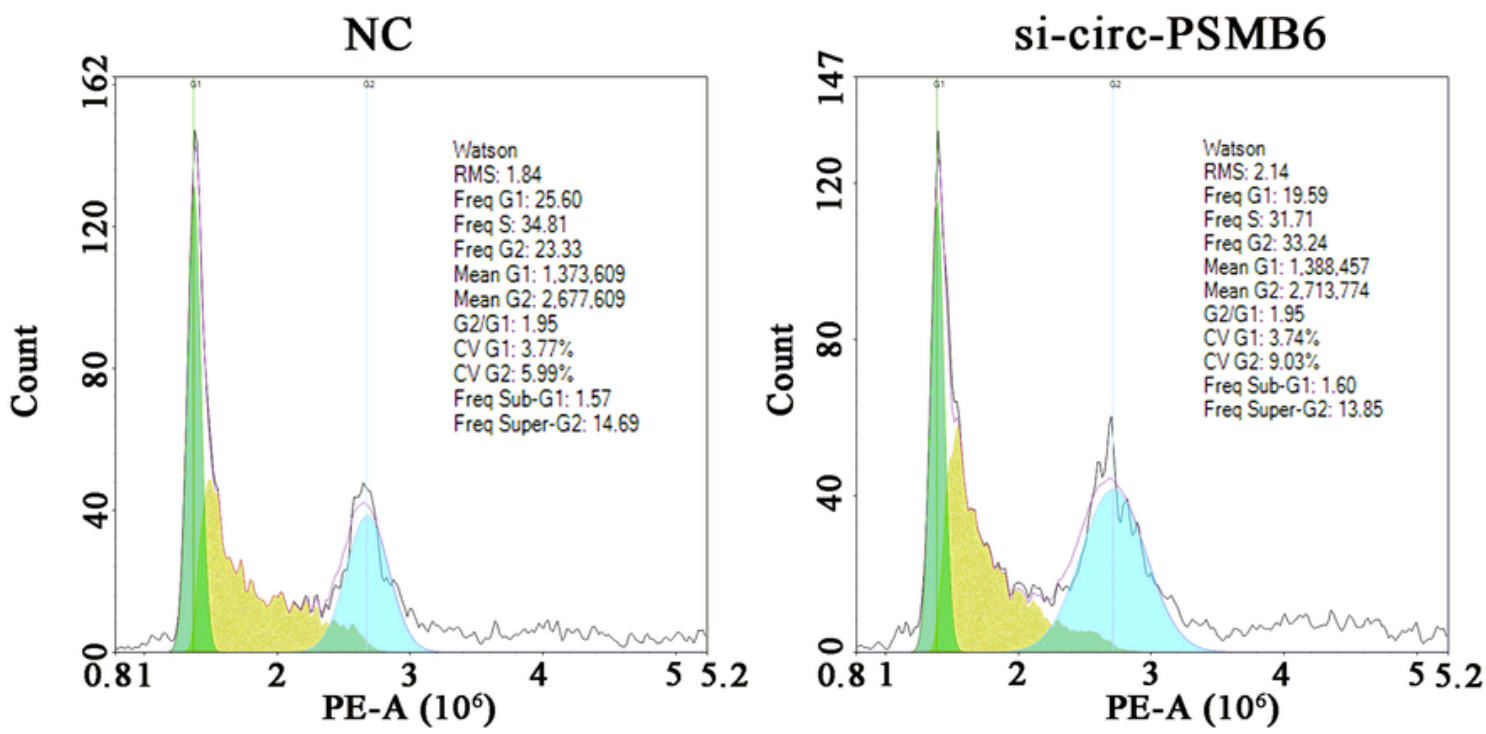

Figure 2

Expression of circ-PSMB6 was increased in NSCLC cells and circ-PSMB6 knockdown arrest cell in G2 phase. (A) The genomic loci of the PSMB6 gene and circ-PSMB6. A red arrow indicates back-splicing. (B) RT-PCR detection of the expression of circ-PSMB6 in A549, PC9, H1299, H1975, H1650, and normal lung epithelial cells BEAS-2B. Data are presented as the mean \pm SD. ${ }^{*} * \mathrm{P}<0.001$ vs. the normal group. (C) RTPCR detection show the expression of circ-PSMB6 after transfected with siRNA against circ-PSMB6 (si- 
circ-PSMB6) or negative control (NC) in both A549 and PC9. (D) Representative results showing the percentage of cells in $\mathrm{G} 1, \mathrm{~S}$, or $\mathrm{G} 2$ phase in $\mathrm{A} 549$ by flow cytometry.

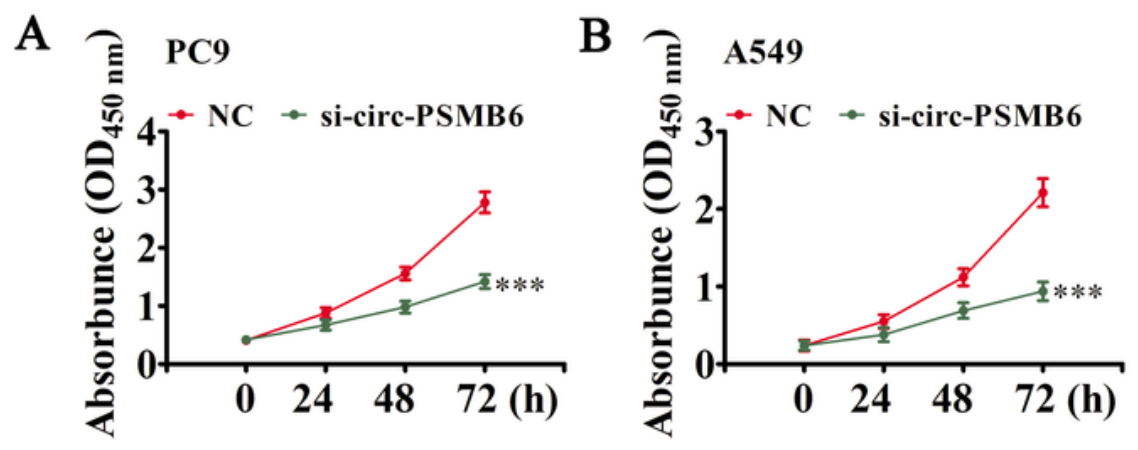

C
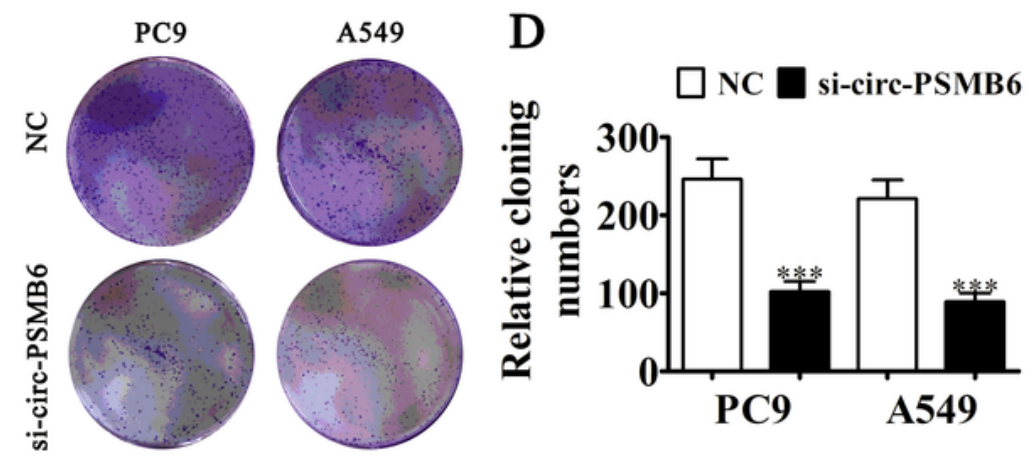

$\mathrm{E}$
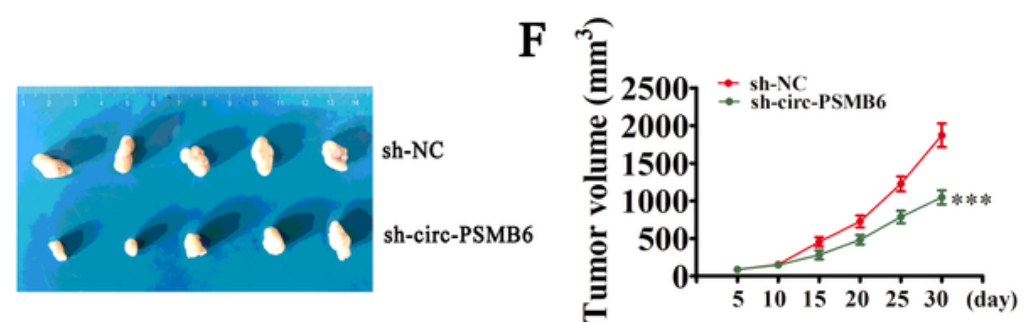

G

$\mathbf{H}$ NC si-circ-PSMB6
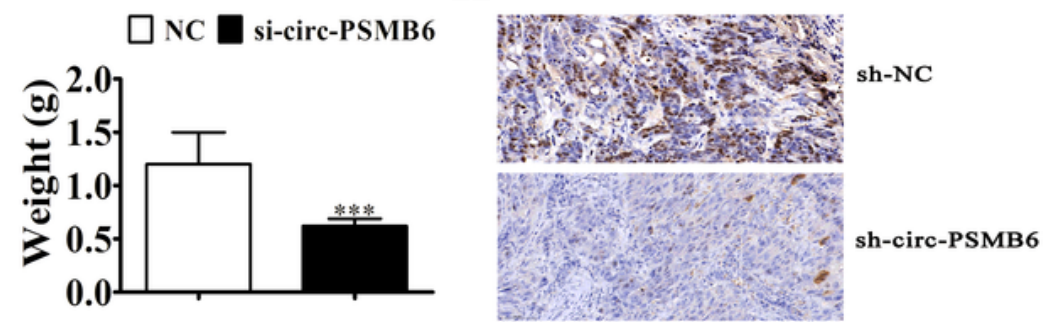

I NC $\square$ si-circ-PSMB6

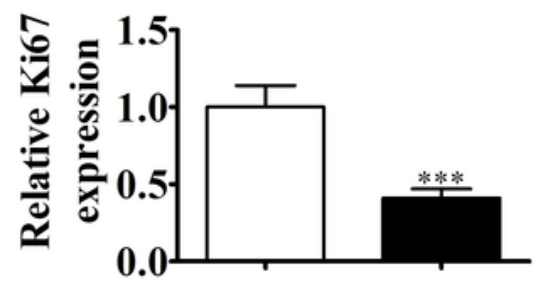

Figure 3

Downregulation circ-PSMB6 inhibit cell proliferation and tumor growth in both in vitro and in vivo experiment. (A and B) Cell proliferation was assessed by CCK-8 assays. Data are presented as means \pm SD. ${ }^{*} *$ P $<0.001$ vs NC. (C and D) PC9 and A549 cell proliferation was assessed by clone formation 
assays. Data are presented as means \pm SD. ${ }^{* \star * P}<0.001$ vs NC. (E) Representative images of nude mouse xenografts after injection of PC9 cells. (F) Tumors growth were measured every 5 days. Data are presented as means \pm SD. ${ }^{* \star *} \mathrm{P}<0.001 \mathrm{vs}$ sh-NC. (G) Tumor weight was measured $30 \mathrm{~d}$ after grafting. Data are presented as means $\pm \mathrm{SD}$. ${ }^{* *} \mathrm{P}<0.001$ vs sh-NC. (H and I) Immunohistochemical staining of Ki67 in tumor specimens from both sh-NC and sh-circ-PSMB6 groups. Data are presented as means \pm SD. $\star * * \mathrm{P}<0.001$ vs sh-NC.

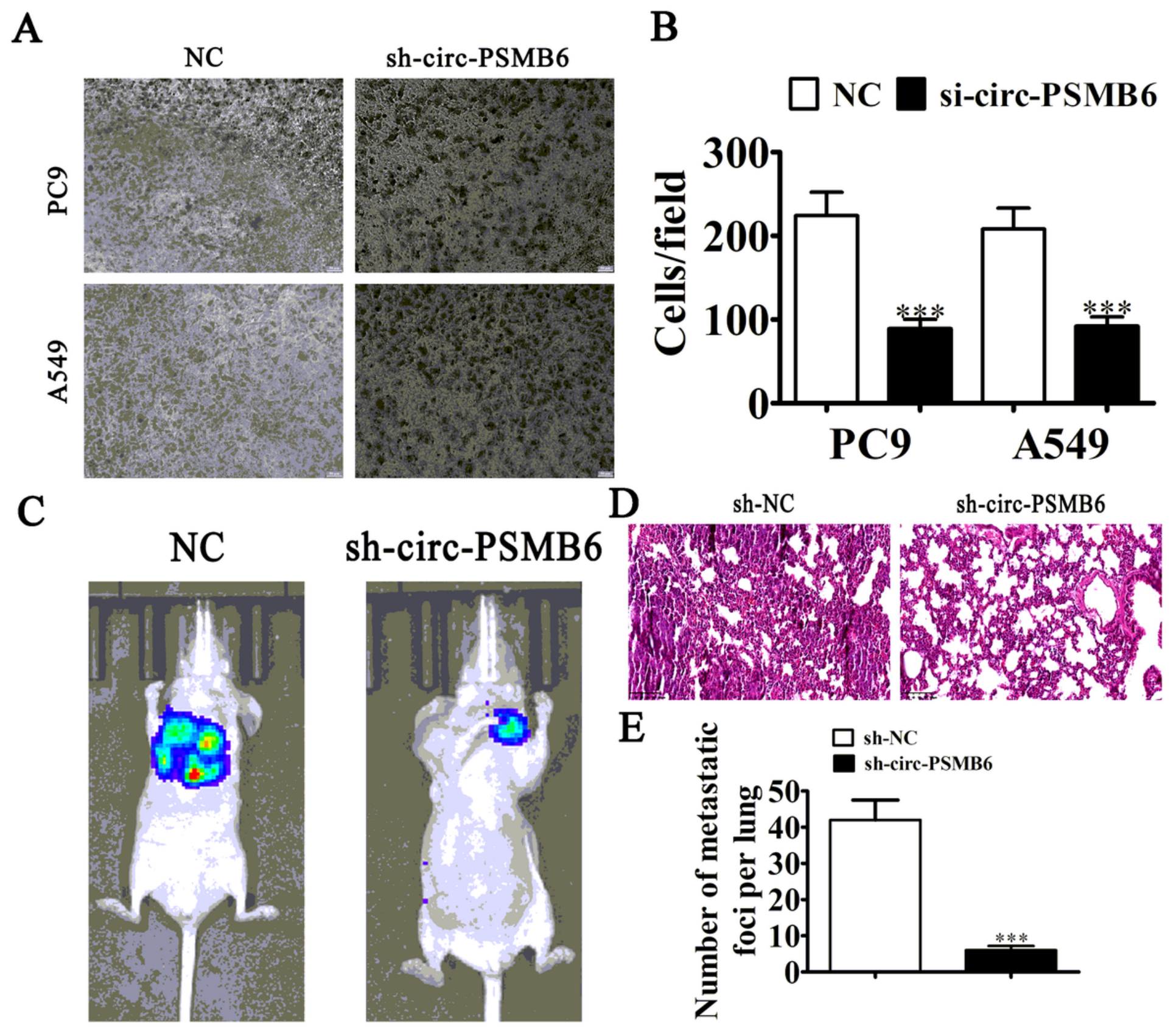

Figure 4

Downregulation circ-PSMB6 inhibit metastasis (A-C) Transwell detection show the migration ability of PC9 and A549 cell with or without circ-PSMB6 silence. Data are presented as means \pm SD. ${ }^{* \star * P}<0.001$ vs NC. (C) Living imaging show the effect of circ-PSMB6 to NSCLC metastasis of PC9 cells after tail 
intravenous injection for 4 weeks. ( $D$ and $E$ ) The numbers of metastatic foci in lung tissues were caculation according to the HE staining. The data are expressed as the mean \pm SD. *** $<<0.001$ vs sh-NC.

A

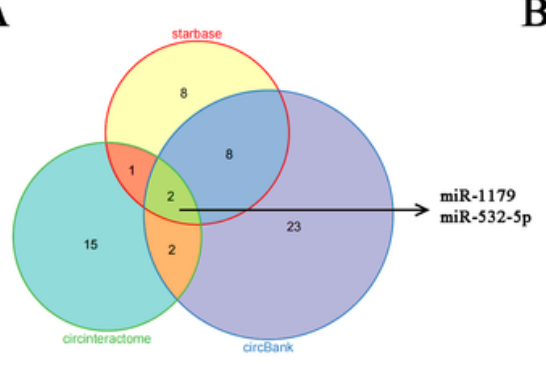

C

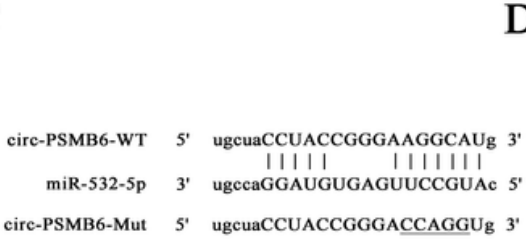

E

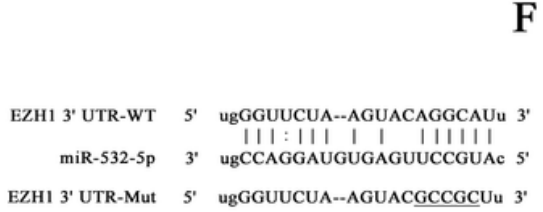

B

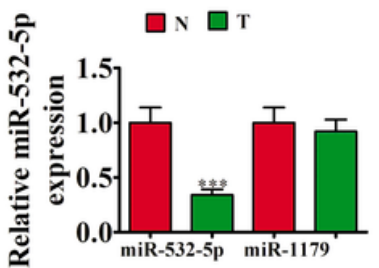

D

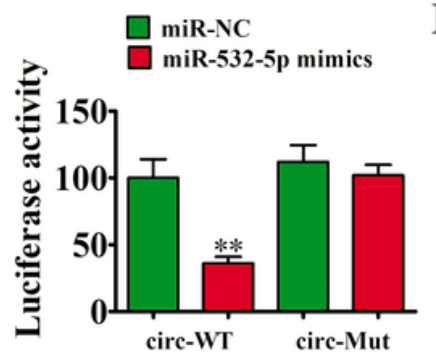

F

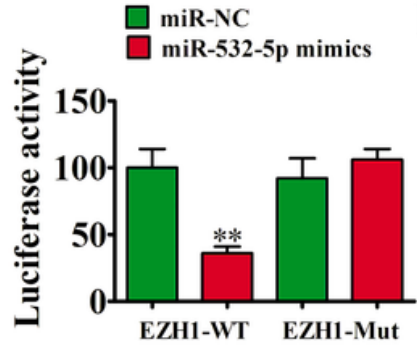

G

PC9

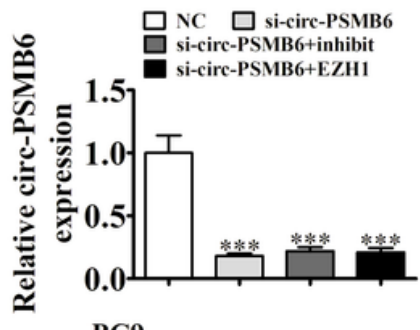

PC9
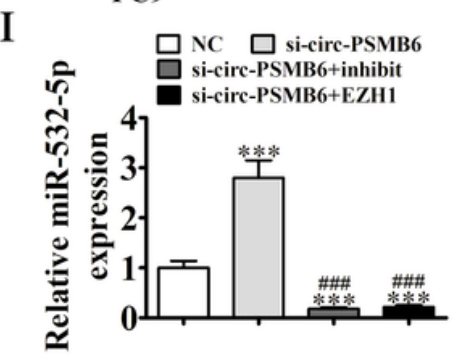

PC9

K

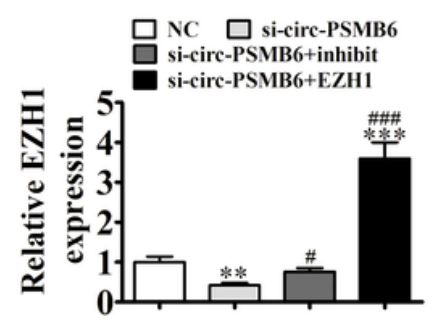

$\mathrm{H}$

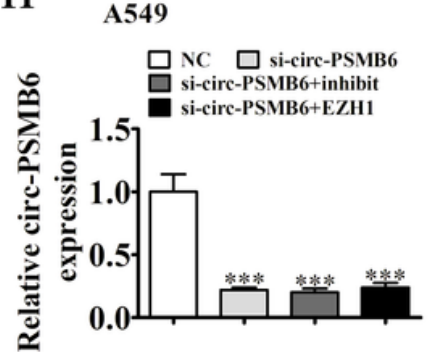

A549

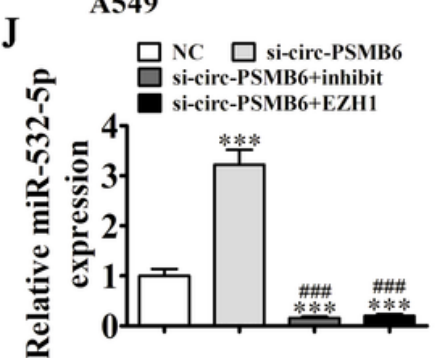

A549

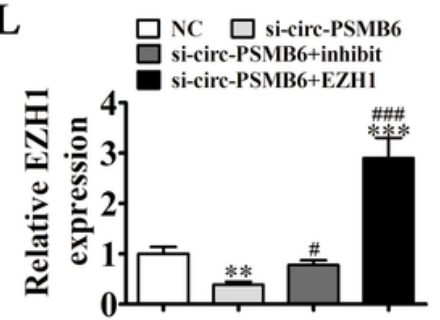

Figure 5

EZH1 and miR-532-5p were the downstream target of circ-PSMB6. (A) bioinformatics analysis and predicted the downstream target of circ-PSMB6 using venn diagram analysis. (B) The luciferase activity of circ-PSMB6 in 293T cells transfected with different miRNA mimics, which are putative binding sites for the circ-PSMB6 sequence. Luciferase activity was normalized by Renilla luciferase activity. Data are presented as mean $\pm \mathrm{SD} ;{ }^{* * *} \mathrm{P}<0.001$ vs N. (C) Prediction of binding sites of miR-532-5p in circ-PSMB6. The MUT version of circ-PSMB6 is presented. (D) Relative luciferase activity determined $48 \mathrm{~h}$ after transfection of 293T cells with miR-532-5p mimic/NC or circ-PSMB6-WT/Mut. Data are presented as means \pm SD. ${ }^{*} \mathrm{P}<0.01$. (E) Prediction of binding sites of miR-532-5p within the $3^{\prime} U T R$ of EZH1. The MUT version of 3'-UTR-EZH1 is shown. (F) Relative luciferase activity determined $48 \mathrm{~h}$ after transfection of 293T cells with miR-532-5p mimic/NC or 3'UTR-EZH1 WT/Mut. Data are presented as means $\pm \mathrm{SD}$. **P< 0.01. (G-L) RT-qPCR detection show the expression of circ-PSMB6, miR-532-5p and EZH1 after transfected with circ-PSMB6 ( $\mathrm{G}$ and $\mathrm{H}$ ), miR-532-5p ( $\mathrm{I}$ and $\mathrm{J}$ ) and EZH1 ( $\mathrm{K}$ and $\mathrm{L}$ ) siRNA or overexpression vector single or combine in both $P C 9$ and A549. Data are presented as mean $\pm S D ; * \star P<0.01,{ }^{\star \star *} P<0.001$ vs NC; \#P<0.05, \#\#\#P<0.001 vs si-circ-PSMB6. 
A

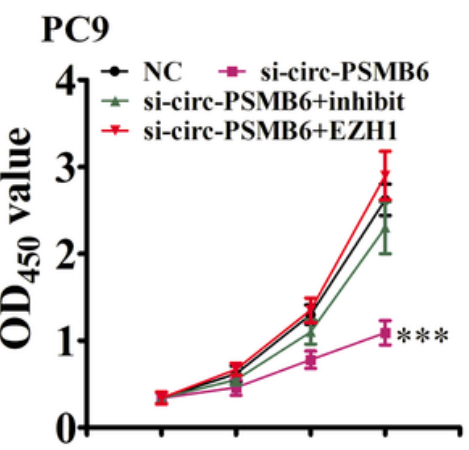

D

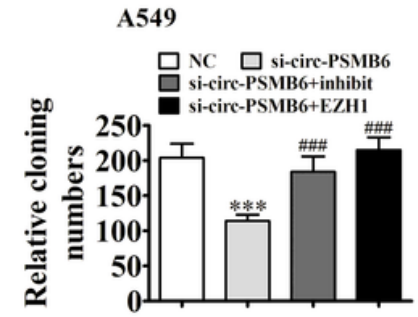

G

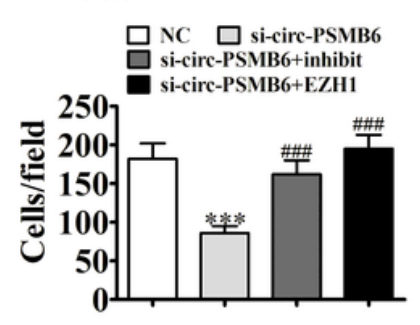

B

A549

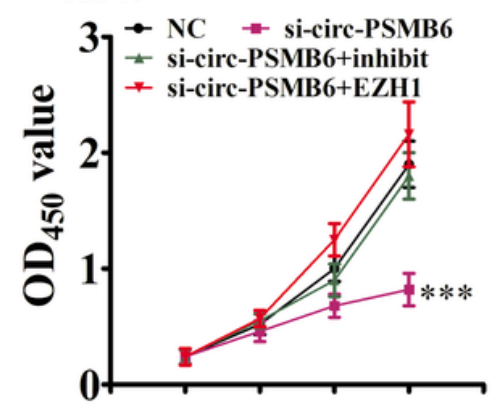

E PC9

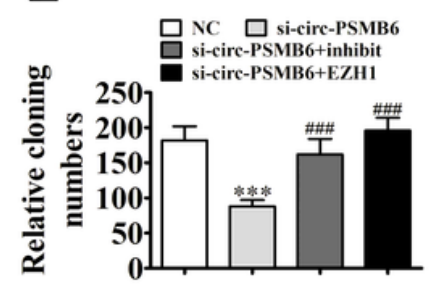

$\mathrm{H}$

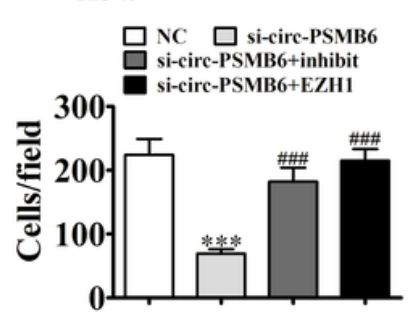

C

si-circ-PSMB6 si-circ-PSMB6

PC9

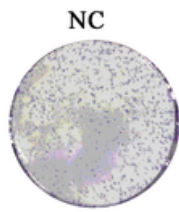

si-circ-PSMB6 +inhibitor

$+\mathrm{EZH} 1$
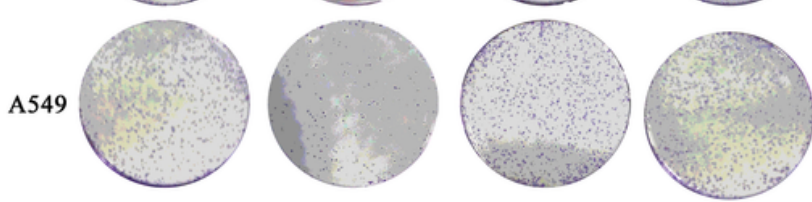

si-circ-PSMB6 si-circ-PSMB6

F

NC

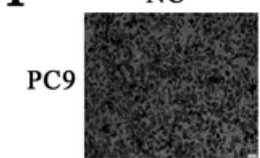

si-circ-PSMB6 +inhibitor

$+\mathrm{EZH} 1$
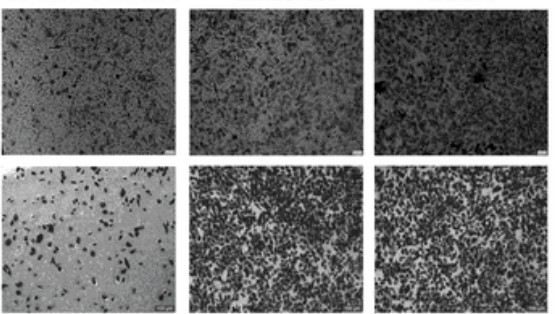

Figure 6

Overexpression of EZH1 or inhibit miR-532-5p reversed NSCLC cells proliferaion and migration after silence circ-PSMB6. (A and B) CCK8 detection show the proliferation ability of PC9 and A549 cells. The data are expressed as the mean $\pm S D$. ${ }^{* *} p<0.001$ vs NC. (C-E) Clone formation assays show the proliferation ability of PC9 and A549 cells. The data are expressed as the mean \pm SD. ${ }^{\star \star *} p<0.001$ vs NC. \#\#\#P<0.001 vs. si-circ-PSMB6. (F-H) Transwell detection show the invasion and migration of PC9 and A549 cells. The data are expressed as the mean \pm SD. ${ }^{\star \star *} \mathrm{p}<0.001$ vs NC. \#\#\#P<0.001 vs. si-circ-PSMB6. 


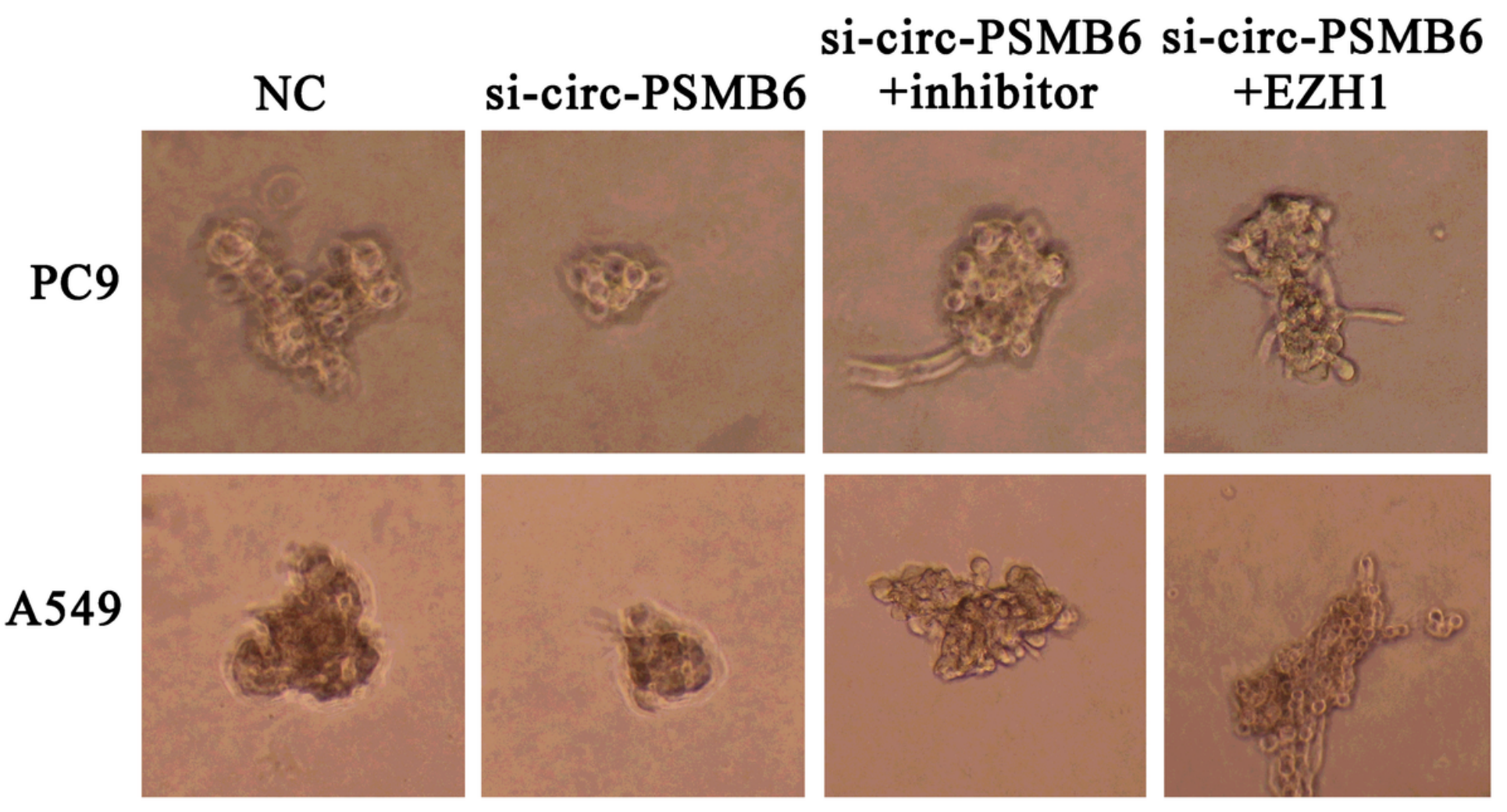

Figure 7

Circ-PSMB6 can influence cancer stem cells different by regulation miR-532-5p/EZH1. Images of tumor sphere formation assays in PC9 and A549 cell. 